International Journal of Bifurcation and Chaos

(c) World Scientific Publishing Company

\title{
Hidden symmetry in a Kuramoto-Sivashinsky initial-boundary value problem
}

\author{
Pietro-Luciano Buono, Lennaert van Veen and Eryn Frawley ${ }^{*}$ \\ Faculty of Science, University of Ontario Institute of Technology, 2000 Simcoe Street North \\ Oshawa, ON L1H7K4, Canada
}

Under review

\begin{abstract}
We investigate the bifurcation structure of the Kuramoto-Sivashinsky equation with homogeneous Dirichlet boundary conditions. Using hidden symmetry principles, based on an extended problem with periodic boundary conditions and $\mathbf{O}(\mathbf{2})$ symmetry, we show that the zero solution exhibits two kinds of pitchfork bifurcations: one that breaks the reflection symmetry of the system with Dirichlet boundary conditions and one that breaks a shift-reflect symmetry of the extended system. Using Lyapunov-Schmidt reduction, we show both to be supercritical. We extend the primary branches by means of numerical continuation, and show that they lose stability in pitchfork, transcritical or Hopf bifurcations. Tracking the corresponding secondary branches reveals an interval of the viscosity parameter in which up to four stable equilibria and time-periodic solutions coexist. Since the study of the extended problem is indispensible for the explanation of the bifurcation structure, the Kuramoto-Sivashinsky problem with Dirichlet boundary conditions provides an elegant manifestation of hidden symmetry.
\end{abstract}

Keywords: Kuramoto-Sivashinsky equation, Lyapunov-Schmidt reduction, equivariant bifurcation, hidden symmetry, Newton-Krylov continuation

\section{Introduction}

In this paper, we explore the bifurcation structure of the Kuramoto-Sivashinsky (KS) equation in one space dimension with Dirichlet Boundary Conditions (BC) as a function of the viscosity parameter. In order to give a fairly complete overview of the bifurcation behaviour close to the leading instability, we use three techniques: an equivariant branching lemma, Lyapunov-Schmidt reduction and numerical continuation of equilibria and time-periodic orbits.

The KS equation was derived from work by Kuramoto [1976] on the Belousov-Zhabotinskii equation and Sivashinsky [1977] on the dynamics of laminar flame fronts. For a summary of the origin, derivation and importance of the KS equation see, e.g., Misbah and Valence [1994] or Wittenberg and Holmes [1999]. Here, we pose it in the following form:

$$
u_{t}+u u_{x}+u_{x x}+\nu u_{x x x x}=0 .
$$

The Dirichlet boundary conditions are

$$
u(-1, t)=u_{x x}(-1, t)=u(1, t)=u_{x x}(1, t)=0 .
$$

*Corresponding author: Eryn.Frawley@uoit.net 
Equation (11) has a rich bifurcation structure, which has been investigated by several authors. Most studies have focused on the case of periodic BC, e.g. Wittenberg and Holmes [1999]; Cvitanovic et al [2010]; Zhang et al. [2011]. In this setting, the KS equations is $\mathbf{O}(\mathbf{2})$ symmetric and the consequences of this symmetry have been explored in detail [Cvitanovic et al, 2010]. There are fewer studies of the KS equation with Dirichlet BC (2). The existence and uniqueness of solutions was established by Galaktionov et al. [2008]. Li and Chen 2001] studied bifurcations from the zero solution with Dirichlet, Neumann and periodic BC. They identify families of bifurcation points for each case and use Lyapunov-Schmidt reduction to determine the criticality of the bifurcating solutions. For the Dirichlet BC case, they identify a family of steady-state bifurcation points with a kernel generated by $\sin (n \pi x)$ for $n \in \mathbb{N}$.

Our first result is the existence of a second family of bifurcation points, with kernel $\cos ([2 n-1] \pi x / 2)$ for $n \in \mathbb{N}$. This includes the leading bifurcation point, i.e. the first instability to occur as the viscosity parameter is reduced from unity, where the zero solution is stable. The KS initial-boundary value problem with Dirichlet $\mathrm{BC}$ has a $\mathbb{Z}_{2}$ symmetry, and the bifurcations with kernel $\cos ([2 n-1] \pi x / 2)$ can readily be explained as breaking this symmetry. Those with kernel $\sin (n \pi x)$ give rise to branches of solutions that lie entirely in the fixed point subspace of the symmetry. Their presence can be explained if we exploit the fact that solutions of the Dirichlet BC case can be seen as restrictions of solutions of the periodic $\mathrm{BC}$ case on an extended domain; this is the phenomenon of hidden symmetry. The extended system has $\mathbf{O}(2)$ symmetry and, accordingly, two-dimensional kernels. Using the structure of the extended problem, we formulate an equivariant branching lemma that determines all bifurcations of the zero state to be of the pitchfork type. In addition, we use Lyapunov-Schmidt reduction to show that all these pitchfork bifurcations are supercritical.

In order to study the nonlinear behaviour of the system, we extend the primary branches by means of numerical continuation. These, too, exhibit pitchfork bifurcations, in addition to transcritical bifurcations, that are neatly explained in terms of the hidden symmetry. All bifurcation points at which primary branches lose stability are identified and the corresponding secondary branches are computed, including two branches of time-periodic solutions. Thus, we are able to give a fairly complete overview of the dynamics of the KS initial-boundary value problem around the first four instabilities of the zero solution.

The paper is organized as follows. Section 2 discusses the linearization at the zero solution and we determine the bifurcation values for the Dirichlet and periodic BC cases. In Section 3, we embed the KS equation with Dirichlet BC on $(-1,1)$ in a periodic BC problem on the doubled-up interval from $(-2,2)$ and derive results from hidden symmetry theory Golubitsky and Stewart, 2002]. This is followed in Section 4 by the computation of the Lyapunov-Schmidt reduction for the bifurcation points at which the kernel is given by $\cos ([2 n-1] \pi x / 2)$. Finally, Section 5 shows the result of numerical continuations of primary and secondary branches of equilibria and time-periodic solutions.

\section{Linearization and Kernels}

It is straightforward to verify that the function $u^{*} \equiv 0$ on $(-1,1)$ is a homogeneous steady-state solution of (1) satisfying (2). The linearization of (11) at $u^{*}$ is

$$
\mathcal{L}(v):=v_{t}+v_{x x}+\nu v_{x x x x}=0
$$

At the homogeneous equilibrium $u^{*}=0$, writing $v(x, t)=(a \cos (k \pi x)+b \sin (k \pi x)) e^{\lambda t}$, the characteristic equation is

$$
\lambda-k^{2} \pi^{2}+\nu k^{4} \pi^{4}=0
$$

and for $\lambda=0$ we have $\nu=(k \pi)^{-2}$. The boundary conditions for $v$ are satisfied for $a=0, k=n$ for $n \in \mathbb{N}$ as shown by Li and Chen [2001], but also for $b=0, k=n-\frac{1}{2}$ where $n \in \mathbb{N}$. The smallest positive value of $k$ is $1 / 2$ and therefore the largest value of $\nu$ at which a bifurcation point arises is

$$
\nu^{*}=\frac{4}{\pi^{2}}
$$

The kernel of $\mathcal{L}$ is one-dimensional at each bifurcation point. For $k=n$, the kernel is spanned by $e_{n}^{\mathrm{o}}(x)=$ $\sin (n \pi x)$ while for $k=n-\frac{1}{2}$, the kernel is spanned by $e_{n}^{\mathrm{e}}(x)=\cos ([2 n-1] \pi x / 2)$. 
We embed the original partial differential equation with Dirichlet boundary conditions on $(-1,1)$ into the domain $(-2,2)$ and we impose periodic boundary conditions

$$
\frac{\partial^{\ell}}{\partial x^{\ell}} u(-2, t)=\frac{\partial^{\ell}}{\partial x^{\ell}} u(2, t), \quad \ell=0,1,2,3 .
$$

For the extended system, at the homogeneous equilibrium $u^{*}=0$, the characteristic equation is unchanged from above and for $\lambda=0$ we still have $\nu=(k \pi)^{-2}$. We obtain the same bifurcation values as the Dirichlet $\mathrm{BC}$ case on the interval $(-1,1)$. The eigenspace is two-dimensional and one can directly check that the kernel is generated by the functions $\{\cos k \pi x, \sin k \pi x\}$. We summarize the results in the following statement.

Proposition 1. For the Dirichlet and periodic $B C$ cases, the linearization $\mathcal{L}$ at the homogeneous zero solution has bifurcation points at $\nu^{*}=(k \pi)^{-2}$ with $k=n$ and $k=n-\frac{1}{2}, n \in \mathbb{N}$. In the Dirichlet $B C$ case, for $k=n$, the kernel is spanned by $e_{n}^{\mathrm{o}}(x)$ while for $k=n-\frac{1}{2}$, the kernel is spanned by $e_{n}^{\mathrm{e}}(x)$. In the periodic $B C$ case, the kernel is spanned by $\{\cos \pi k x, \sin \pi k x\}$ both for $k=n$ and $k=n-\frac{1}{2}$.

In the next section, we explore the relationship between the solutions of the Dirichlet BC problem on $(-1,1)$ and the periodic BC case on $(-2,2)$ defined above.

\section{Hidden symmetry}

We now discuss the hidden symmetry properties of (11) with Dirichlet boundary conditions (2) and in particular, show that all steady-state bifurcations from the trivial solution must be pitchfork bifurcations.

It is well-known Golubitsky and Stewart, 2002], that an equation such as (1) with boundary conditions (3) is symmetric with respect to a group generated by reflections and translations. In particular, if $u(x, t)$ is a solution then

$$
\kappa . u(x, t)=-u(-x, t) \quad \text { and } \quad \theta . u(x, t)=u(x-\theta, t), \quad \theta \in(-2,2]
$$

are also solutions. In particular, $\theta^{-1} \kappa=\kappa \theta$ for all $\theta \in(-2,2]$. The group generated by $\langle\kappa, \theta\rangle$ is isomorphic to the group $\mathbf{O}(\mathbf{2})$.

We drop the dependence on $t$ and define the space

$$
X_{\text {per }}=\left\{u \in C^{4}([-2,2], \mathbb{R}) \mid u \text { satisfies boundary conditions (3) }\right\} .
$$

The group $\Gamma=\mathbf{O}(2)$ acts on this space of functions by the formulae given in (44).

At the bifurcation points $\left(u^{*}, \nu_{k}^{*}\right)=\left(0,(k \pi)^{-2}\right)$ for $k=n-\frac{1}{2}$ and $k=n$ with $n \in \mathbb{N}$, the kernel of the linearization at $\nu_{k}^{*}$ is two-dimensional and generated by the functions $\{\cos k \pi x, \sin k \pi x\}$ as noted in Proposition 1.

The kernel of the linearization is an absolutely irreducible representation of $\mathbf{O}(\mathbf{2})$. From the Equivariant Branching Lemma Golubitsky et al., 1988], a branch of equilibrium solutions for each isotropy subgroup with fixed point subspace of dimension one bifurcates from $\left(u^{*}, \nu_{k}^{*}\right)$. The two-dimensional $\mathbf{O}(\mathbf{2})$ irreducible representation spanned by $\{\cos k \pi x, \sin k \pi x\}$ has a family of isotropy subgroups $\Sigma_{k, \theta}=\left\langle\theta^{-1} \kappa \theta, 2 / k\right\rangle$ with $2 / k$ a translation, each isomorphic to $\mathbf{D}_{2 k}$, where $\theta \in[-2 / k, 2 / k) . \Sigma_{k, \theta}$ fixes the one-dimensional subspace spanned by $\sin k \pi \theta \cos k \pi x+\cos k \pi \theta \sin k \pi x=\sin (k \pi(x+\theta))$. Note that the translation by $1 / k$ acts by -1 on $\operatorname{Fix}\left(\Sigma_{k, \theta}\right)$ for all $\theta$ :

$$
(1 / k) \cdot \sin (k \pi(x+\theta))=\sin (k \pi(x+\theta)) \cos (\pi)=-\sin (k \pi(x+\theta)) .
$$

Finally, one can verify that $\operatorname{Fix}\left(\Sigma_{k, 1 / 2 k}\right)=\operatorname{Fix}\left(\Sigma_{k,-1 / 2 k}\right)=\operatorname{span}\{\cos k \pi x\}$ and

$$
\operatorname{Fix}\left(\Sigma_{k, 1 / k}\right)=\operatorname{Fix}\left(\Sigma_{k,-1 / k}\right)=\operatorname{Fix}\left(\Sigma_{k, 2 / k}\right)=\operatorname{Fix}\left(\Sigma_{k, 0}\right)=\operatorname{span}\{\sin k \pi x\}
$$

Proposition 2. The periodic $B C$ problem has $\mathbf{O}(\mathbf{2})$ steady-state bifurcation points at $\left(0, \nu_{k}^{*}\right)$ where $\nu_{k}^{*}=$ $(k \pi)^{-2}$ for $k=n-1 / 2$ and $k=n$ with $n \in \mathbb{N}$. An $\mathbf{O}(\mathbf{2})$-orbit of steady-state solutions with isotropy subgroup $\Sigma_{k, \theta}$ conjugate to $\boldsymbol{D}_{2 k}$ bifurcates from $\left(0, \nu_{k}^{*}\right)$ where solutions with the same isotropy subgroup come in pairs related by the translation element $\theta_{1 / k}$. 
Remark 3.1. At bifurcation points $\nu_{k}^{*}$ for $k=n-1 / 2$, the symmetry group $\Sigma_{k, \theta}$ has cyclic symmetry of order $2 n-1$ while for $k=n$ the cyclic symmetry is of order $2 n$.

We now present the connections between the solutions of the Dirichlet BC case with the periodic BC case, We begin with this result concerning the regularity of solutions related by hidden symmetry. The proof describes how equilibrium solutions are extended from the smaller to the larger domain. The proof follows the one given in Golubitsky and Stewart [2002] for reaction-diffusion equations.

Lemma 1. Smooth equilibrium solutions of the Dirichlet $B C$ problem on $[-1,1]$ extend to smooth equilibrium solutions of the periodic $B C$ problem on $[-2,2]$.

Proof. Let $u(x)$ be an equilibrium solution of (1) with Dirichlet BC on $[-1,1]$; the time-dependence of $u$ is suppressed as it is not needed. Extend $u(x)$ to $\hat{u}(x)$ defined by

$$
\hat{u}(x)=\left\{\begin{array}{cc}
-u(-2-x), & x \in[-2,-1] \\
u(x), & x \in[-1,1] \\
-u(2-x), & x \in[1,2] .
\end{array}\right.
$$

The function $\hat{u}$ is constructed from $u$ by extending in an odd way with respect to $x=1$ the portion defined over $[0,1]$ to $[1,2]$. Similarly, extending in an odd fashion the portion over $[-1,0]$ with respect to $x=-1$ to $[-2,-1]$.

Then, $\hat{u}(x)$ satisfies (1+2) automatically on $[-1,1]$ and we show the proof for the portion of $\hat{u}$ defined on $[-2,-1]$. For $x \in[-2,-1]$, let $y=-2-x$, then $y \in[0,1]$ and the partial derivatives with respect to $x$ become partial derivatives with respect to $y$ so that

$$
\hat{u}(x) \hat{u}_{x}(x)+\hat{u}_{x x}(x)+\nu \hat{u}_{x x x x}(x)=u(y, t) u_{y}(y)+u_{y y}(y)+\nu u_{y y y y}(y)=0 .
$$

An identical computation holds for the interval [1,2]. It is straightforward to check that the periodic boundary conditions are satisfied. The smoothness needs to be verified only at $x= \pm 1$. We do the case at $x=1$, the computations at $x=-1$ are similar and omitted. We use the notation $1^{-}$and $1^{+}$to denote the left and right limits at $x=1$. By construction of $\hat{u}$, we have $\hat{u}\left(1^{-}\right)=\hat{u}\left(1^{+}\right)$. Note that for $x \in(1,2)$ the $k^{\text {th }}$ derivative satisfies $\hat{u}^{(k)}(x)=(-1)^{k+1} u^{(k)}(2-x)$. Thus, $\hat{u}^{(2 k+1)}\left(1^{+}\right)=u^{(2 k+1)}\left(1^{-}\right)=\hat{u}^{(2 k+1)}\left(1^{-}\right)$for all $k \in \mathbb{N}$. Now, $\hat{u}^{\prime \prime}\left(1^{+}\right)=-u^{\prime \prime}\left(1^{-}\right)=\hat{u}^{\prime \prime}\left(1^{-}\right)$, but we know that $u^{\prime \prime}(1)=0$ because $u$ satisfies the Dirichlet BC. Finally, using (2) we have

$$
u^{(4)}( \pm 1)=-\nu^{-1}\left(u( \pm 1) u_{x}( \pm 1)+u_{x x}( \pm 1)\right)=0
$$

and this implies $\hat{u}^{(4)}\left(1^{-}\right)=\hat{u}^{(4)}\left(1^{+}\right)=0$. We show by induction that $u^{(2 k)}( \pm 1)=0$ with $k \geq 3$. For $k=3$,

$$
u^{(6)}( \pm 1)=-\nu^{-1}\left(3 u_{x}( \pm 1) u_{x x}( \pm 1)+u_{x x x x}( \pm 1)\right)=0 .
$$

For $u^{(2 k)}(x)$, one can verify that the expression for this derivative depends only on terms of the form $u^{\ell} u^{n}$ where $\ell$ is odd and $n$ is even and the term $u_{x x}$ obtained from substituting for $u^{(4)}(x)$. But the second derivative of terms such as $u^{\ell} u^{n}$ is in terms of a sum of terms of the same form. Thus, evaluating at \pm 1 , those all vanish, and we know $u_{x x}( \pm 1)=0$, thus $u^{(2 k)}( \pm 1)=0$. Therefore, $\hat{u}$ is smooth.

We can characterize using the symmetry reflection the solutions of the periodic BC problem which satisfy the Dirichlet BC.

Lemma 2. Let $v$ be a steady-state solution of the extended periodic BC problem on $[-2,2]$. Then, $v=\hat{u}$ where $u$ satisfies the Dirichlet BC problem if and only if $v \in F i x\left((1)^{-1} \mathbb{Z}_{2}(\kappa)(1)\right)$.

Proof. Suppose $v=\hat{u}$ satisfies the periodic BC problem. Then, by construction of $\hat{u}$, we have

$$
v(x-1)=\left\{\begin{array}{cc}
-u(1-x), & x \in[-1,0] \\
u(x-1), & x \in[0,2] \\
-u(1-x), & x \in[-2,-1]
\end{array}\right.
$$


and so $v(x-1)$ is an odd function, that is, $v(x-1) \in \operatorname{Fix}\left(\mathbb{Z}_{2}(\kappa)\right)$. This means $v(x) \in \operatorname{Fix}\left(1^{-1} \mathbb{Z}_{2}(\kappa) 1\right)$. The opposite implication is straightforward because elements in the subspace $\operatorname{Fix}\left(1^{-1} \mathbb{Z}_{2}(\kappa) 1\right)$ automatically satisfy the Dirichlet BC.

The main result of this section concerns the steady-state bifurcation points of (11) and (2).

Theorem 1. The Dirichlet BC problem (1) and (2) has, generically, pitchfork steady-state bifurcation points at $\left(0, \nu_{k}^{*}\right)$ for $k=n-1 / 2$ and $k=n$ for $n \in \mathbb{N}$. If $k=n-1 / 2$, the pair of bifurcating solutions are on the same $\kappa$ orbit. If $k=n$, the pair of bifurcating solutions are fixed by $\mathbb{Z}_{2}(\kappa)$, and therefore not related by symmetry.

Proof. At the bifurcation points $\left(0, \nu_{k}^{*}\right)$ for $k=n-1 / 2$ in the periodic BC case, there are two branches of steady-state solutions (a $1 / 2 k$ group orbit) with isotropy subgroup $\Sigma_{k, 1 / 2 k} \supset(1)^{-1} \mathbb{Z}_{2}(\kappa)(1)$. By Lemma 2 , the pair of bifurcating solutions also satisfy the Dirichlet BC problem (1) and (2). We now show that for the periodic $\mathrm{BC}$ problem, $1 / k$ acts on $\operatorname{Fix}\left(\Sigma_{k, 1 / 2 k}\right)$ in the same way as $\kappa$. Let $u(x) \in \operatorname{Fix}\left(\Sigma_{k, 1 / 2 k}\right)$ and recall that $(1 / 2 k) \cdot u(x)$ is fixed by $\kappa$. Then, using the non-commutativity relationship of $\theta$ and $\kappa$,

$$
(\kappa(1 / k)) \cdot u(x)=(\kappa(1 / 2 k)) \cdot u(x-1 / 2 k)=(1 / 2 k)^{-1} \kappa \cdot u(x-1 / 2 k)=(1 / 2 k)^{-1} u(x-1 / 2 k)=u(x)
$$

which indeed means $\theta_{1 / k} \cdot u(x)=-u(-x)=\kappa \cdot u(x)$. Therefore, the pair of bifurcating solutions is related by the $\kappa$ symmetry which is the only remaining symmetry of the Dirichlet BC problem.

Consider the $k=n$ bifurcation points. The kernel at those bifurcating points of the periodic BC problem restricted to $\operatorname{Fix}\left(\Sigma_{k, 0}\right)$ has a pair of bifurcating branches of steady-state solutions satisfying the the Dirichlet BC problem. But $\mathbb{Z}_{2}(\kappa) \subset \Sigma_{k, 0}$ and so the bifurcating pair for the Dirichlet BC problem are not related by a symmetry.

Remark 3.2. For the cases $k=n \in \mathbb{Z}$ of the Dirichlet BC problem, Li and Chen [Li and Chen, 2001] show that the cubic coefficient obtained via Lyapunov-Schmidt reduction is negative therefore proving that the pitchfork bifurcations are supercritical. It is also shown in $\mathrm{Li}$ and Chen, 2001] that the periodic BC case for the cases $k=n$ restricted to the fixed point subspaces with kernels consisting of $\sin (k \pi x)$ and $\cos (k \pi x)$ are also supercritical. As this case has in fact $\mathbf{O}(\mathbf{2})$-symmetry, it is only necessary to verify one of them to determine the supercritical nature of the bifurcation of the $\mathbf{O}(\mathbf{2})$ group orbit.

In the next section, we complete the analysis by using the Lyapunov-Schmidt reduction to determine the criticality at the bifurcation points with $k=n-1 / 2$ for the Dirichlet BC case.

We conclude this section by looking at secondary bifurcations, that is, bifurcations from the primary branches of bifurcation emanating from the $u^{*}=0$ steady-state. We use again the periodic BC case to obtain the results and we separate the $k=n-1 / 2$ and $k=n$ cases.

We begin with the $k=n-1 / 2$ case and recall from Remark 3.1 that the branches of steady-states bifurcating at $\nu_{k}^{*}$ have isotropy subgroup $\Sigma_{k, \theta} \simeq \mathbf{D}_{2 n-1}$ Standard results from equivariant bifurcation theory with dihedral symmetry Golubitsky et al., 1988] state that generic symmetry-breaking bifurcations from a steady-state with isotropy subgroup $\mathbf{D}_{2 n-1}$ lead to a unique (up to conjugacy) branch of steady-state solutions with isotropy subgroup given by the reflection symmetry. We focus on the isotropy subgroup $\Sigma_{k, 1 / 2 k}$ and so the isotropy subgroup of a bifurcating branch from the $\Sigma_{k, 1 /(2 k)}$ branch is $\mathbb{Z}_{2}\left((1 /(2 k))^{-1} \kappa(1 /(2 k))\right)$ and there are no other element of $\mathbf{O}(\mathbf{2})$ leaving $\operatorname{Fix}\left(\mathbb{Z}_{2}\left((1 /(2 k))^{-1} \kappa(1 /(2 k))\right)\right.$ invariant; that is, $\mathbb{Z}_{2}\left((1 /(2 k))^{-1} \kappa(1 /(2 k))\right)$ is its own normalizer subgroup. From Theorem 1 , to the branch of steady-state solutions with isotropy subgroup $\Sigma_{k, 1 /(2 k)}$ there corresponds a branch of steady-state solutions of the Dirichlet BC problem. Therefore, if the steady-state solution branch with $\Sigma_{k, 1 / 2 k}$ isotropy subgroup has a symmetry-breaking steady-state bifurcation to a branch of solutions which also satisfy the Dirichlet $\mathrm{BC}$, then, generically, it appears as a transcritical bifurcation in the Dirichlet BC problem.

The case $k=n$ leads to isotropy subgroups $\Sigma_{k, \theta} \simeq \mathbf{D}_{2 n}$ and generic steady-state bifurcation results with dihedral symmetry $\mathbf{D}_{2 n}$ leads to pitchfork bifurcations because the normalizer subgroup of the $\mathbb{Z}_{2}$ symmetric branches acts by -1 on the fixed point subspaces. Focusing on the isotropy subgroup $\Sigma_{k, 0}$, we 
know this branch of steady-state solutions also satisfies the Dirichlet BC case and if it has a symmetrybreaking bifurcation which also satisfies the Dirichlet BC, then it appears as a pitchfork bifurcation in the Dirichlet BC problem.

\section{Lyapunov-Schmidt reduction}

We now determine the criticality of the primary branches of steady-states bifurcating from the bifurcation points at $\nu_{k}^{*}$ for $k=n-1 / 2$. We use the Lyapunov-Schmidt reduction to perform this analysis. For the Lyapunov-Schmidt reduction process below, it is convenient to rewrite the time-independent part of the equation abstractly as follows. We define the function space

$$
X=\left\{u \in C^{4}[-1,1] \mid u(-1)=u(1)=u^{\prime \prime}(-1)=u^{\prime \prime}(1)=0\right\},
$$

and let $Y=C^{0}[-1,1]$. The two spaces share the inner product defined as

$$
\langle u, v\rangle=\int_{-1}^{1} u(\xi) v(\xi) d \zeta .
$$

Theorem 2. For all the bifurcating points $\nu_{k}^{*}$ with $k=n-1 / 2$ with $n \in \mathbb{N}$, the branches of steady-states bifurcate supercritically. For the largest bifurcation point $\nu_{1 / 2}^{*}$, the steady-state solutions bifurcating are asymptotically stable.

Proof. We rewrite the steady-state Kuramoto-Sivashinsky equation using the mapping $\Phi: X \times \mathbb{R} \rightarrow Y$ as

$$
\Phi(u, \nu)=u u_{x}+u_{x x}+\nu u_{x x x x}=0 .
$$

We define the linear operator $L_{0}: X \times \mathbb{R} \mapsto Y$ as follows

$$
L_{0}=d \Phi\left(0, \nu_{k}^{*}\right)=\nu_{k}^{*} \frac{d^{4}}{d \xi^{4}}+\frac{d^{2}}{d \xi^{2}}
$$

and we remove on purpose the dependence of this linear operator on $k$ to lighten the notation. $L_{0}: X \rightarrow Y$ is self-adjoint because of the boundary conditions defining $X$. Furthermore, $\operatorname{ker} L_{0}=\operatorname{span}\{e\}$, where $e=e_{n}^{\mathrm{e}}(\xi)$. To keep the notation as simple as possible below, we let $\beta=\frac{\pi}{2}(2 n-1)$. The subspaces ker $L_{0}$ and range $L_{0}$ enable us to decompose the spaces $X$ and $Y$ :

$$
X=\operatorname{ker} L_{0} \oplus\left(\operatorname{ker} L_{0}\right)^{\perp} \quad \text { and } \quad Y=\left(\operatorname{range} L_{0}\right)^{\perp} \oplus \operatorname{range} L_{0} .
$$

Notice that because $L_{0}$ is self-adjoint, then ker $L_{0}=\left(\text { range } L_{0}\right)^{\perp}$. We can now define $P$ as the orthogonal projection from $Y$ onto range $L_{0}$. Let $u \in X$, then the decomposition of $X$ leads to $u=y e+w$, where $y \in \mathbb{R}$ is the coordinate on the kernel and $w \in\left(\operatorname{ker} L_{0}\right)^{\perp}$. The projection $P$ can be applied to $\Phi$ to decompose it in two separate parts, namely

$$
G(y, w, \nu):=P \Phi(y e+w, \nu)=0 \quad \text { and } \quad(I-P) \Phi(y e+w, \nu)=0 .
$$

Because $G\left(0,0, \nu_{k}^{*}\right)=0$ and $d G\left(0,0, \nu_{k}^{*}\right)$ is surjective on range $L_{0}$, by the implicit function theorem, there exists a unique function $w=w(y, \nu)$ with $w\left(0, \nu_{k}^{*}\right)=0$ such that $G(y, w(y, \nu), \nu) \equiv 0$. The equation $(I-P) \Phi(y e+w(y, \nu), \nu)=0$ is transformed by taking the inner product with $e$, from which we obtain

$$
g(y, \nu)=\langle e, \Phi(y e+w(y, \nu), \nu)\rangle=0
$$

since $P \Phi$ is orthogonal to $e$. We want to find an approximation of the system defined by $g(y, \nu)$ at the point $\left(0, \nu_{k}^{*}\right)$, so it is necessary to take the partial derivatives of $g(y, \nu)$. Only the first three derivatives in terms of $y$, the first derivative with respect to $\nu$, and the first mixed derivative are required in this case. By using the Taylor expansion of this system, we obtain the following derivatives:

$$
\begin{aligned}
g_{y} & =\left\langle e, d \Phi\left(0, \nu_{k}^{*}\right)\left(e+w_{y}\right)\right\rangle \\
g_{y^{2}} & =\left\langle e, d \Phi\left(0, \nu_{k}^{*}\right)\left(w_{y^{2}}\right)+d^{2} \Phi\left(0, \nu_{k}^{*}\right)\left(e+w_{y}, e+w_{y}\right)\right\rangle \\
g_{y^{3}} & =\left\langle e, d \Phi\left(0, \nu_{k}^{*}\right)\left(w_{y^{3}}\right)+3 d^{2} \Phi\left(0, \nu_{k}^{*}\right)\left(e+w_{y}, w_{y^{2}}\right)+d^{3} \Phi\left(0, \nu_{k}^{*}\right)\left(e+w_{y}, e+w_{y}, e+w_{y}\right)\right\rangle \\
g_{\nu} & =\left\langle e, d \Phi\left(0, \nu_{k}^{*}\right)\left(w_{\nu}\right)+\Phi_{\nu}\left(0, \nu_{k}^{*}\right)\right\rangle \\
g_{y \nu} & =\left\langle e, d \Phi_{\nu}\left(0, \nu_{k}^{*}\right)\left(e+w_{y}\right)+d \Phi\left(0, \nu_{k}^{*}\right)\left(w_{y \nu}\right)+d^{2} \Phi\left(0, \nu_{k}^{*}\right)\left(e+w_{y}, w_{\nu}\right)\right\rangle
\end{aligned}
$$


We obtain the explicit values of the first few derivatives of $w$ with respect to $y$ and $\nu$ from the equation $G(y, w(y, \nu), \nu) \equiv 0$, those are:

$$
\begin{aligned}
& 0=\operatorname{Pd} \Phi\left(0, \nu_{k}^{*}\right)\left(e+w_{y}\right) \\
& 0=\operatorname{Pd} \Phi\left(0, \nu_{k}^{*}\right)\left(w_{y^{2}}\right)+P d^{2} \Phi\left(0, \nu_{k}^{*}\right)\left(e+w_{y}, e+w_{y}\right) \\
& 0=\operatorname{Pd} \Phi\left(0, \nu_{k}^{*}\right)\left(w_{y^{3}}\right)+P 3 d^{2} \Phi\left(0, \nu_{k}^{*}\right)\left(e+w_{y}, w_{y^{2}}\right)+P d^{3} \Phi\left(0, \nu_{k}^{*}\right)\left(e+w_{y}, e+w_{y}, e+w_{y}\right) \\
& 0=\operatorname{Pd} \Phi\left(0, \nu_{k}^{*}\right)\left(w_{\nu}\right)+P \Phi_{\nu}\left(0, \nu_{k}^{*}\right) \\
& 0=\operatorname{Pd} \Phi_{\nu}\left(0, \nu_{k}^{*}\right)\left(e+w_{y}\right)+\operatorname{Pd} \Phi\left(0, \nu_{k}^{*}\right)\left(w_{y \nu}\right)+P d^{2} \Phi\left(0, \nu_{k}^{*}\right)\left(e+w_{y}, w_{\nu}\right)
\end{aligned}
$$

For both sets of equations (6) and (17), the derivatives of $w$ are evaluated at $\left(0, \nu_{k}^{*}\right)$ and we now obtain explicit expressions for them. Recall that $L_{0}=d \Phi\left(0, \nu_{k}^{*}\right)$ and $P L_{0}=L_{0}$. We begin with

$$
0=\operatorname{Pd} \Phi\left(0, \nu_{k}^{*}\right)\left(e+w_{y}\right)=L_{0}(e)+L_{0}\left(w_{y}\right)=L_{0} w_{y} .
$$

But $w_{y}\left(0, \nu_{k}^{*}\right) \in\left(\operatorname{ker} L_{0}\right)^{\perp}$ because $w(y, \nu) \in\left(\operatorname{ker} L_{0}\right)^{\perp}$. By the invertibility of $L_{0}$ on $\left(\operatorname{ker} L_{0}\right)^{\perp}$ we have $w_{y}\left(0, \nu_{k}^{*}\right)=0$ and so $g_{y}\left(0, \nu_{k}^{*}\right)=0$ from the first equation in (6).

From a Taylor expansion of equation (15), one can show that $d^{2} \Phi\left(0, \nu_{k}^{*}\right)\left(\zeta_{1}, \zeta_{2}\right)=\frac{d}{d x}\left(\zeta_{1} \zeta_{2}\right)$ where $\zeta_{1}, \zeta_{2}$ are functions in the tangent space of $X$ at $\left(0, \nu_{k}^{*}\right)$. This is applied to $P d \Phi\left(0, \nu_{k}^{*}\right)\left(w_{y^{2}}\right)+P d^{2} \Phi\left(0, \nu_{k}^{*}\right)(e+$ $\left.w_{y}, e+w_{y}\right)=0$. Because $w_{y}=0$, this implies

$$
P L_{0} w_{y^{2}}\left(0, \nu_{k}^{*}\right)+P\left[\left(e^{2}\right)^{\prime}\right]=0,
$$

where $\left(e^{2}\right)^{\prime}=\left(\cos ^{2}(\beta \xi)\right)^{\prime}=-\beta \sin (2 \beta \xi)$ from which we get

$$
w_{y^{2}}\left(0, \nu_{k}^{*}\right)=\beta L_{0}^{-1} \sin (2 \beta \xi)=\frac{\beta}{(2 \beta)^{2}\left(\nu_{k}^{*}(2 \beta)^{2}-1\right)} \sin (2 \beta \xi)=\frac{1}{6 \pi(2 n-1)} \sin (2 \beta \xi) .
$$

Note that $\sin$ is an eigenfunction of $L_{0}$ and so the $L_{0}^{-1} \sin (2 \beta \xi)$ computation is obtained by inverting the corresponding eigenvalue. Now, $g_{y^{2}}=\left\langle e, d \Phi\left(0, \nu_{k}^{*}\right)\left(w_{y^{2}}\right)+d^{2} \Phi\left(0, \nu_{k}^{*}\right)\left(e+w_{y}, e+w_{y}\right)\right\rangle$ and by substitution of both $L_{0}$ and $\left(e^{2}\right)^{\prime}$ we obtain

$$
g_{y^{2}}\left(0, \nu_{k}^{*}\right)=\left\langle e, L_{0} w_{y^{2}}\left(0, \nu_{k}^{*}\right)+\left(e^{2}\right)^{\prime}\right\rangle=0
$$

because $\left\langle e,\left(e^{2}\right)^{\prime}\right\rangle=0$ by oddness of the integrand and $\left\langle e, L_{0} w_{y^{2}}\right\rangle$ vanishes by orthogonality of cos and $\sin$. Notice that in the expression for $g_{y_{3}}$ from (6), we have $\left\langle e, L_{0}\left(w_{y^{3}}\right)\right\rangle=0$ by orthogonality of ker $L_{0}$ with range $L_{0}$. By Taylor expansion of $\Phi$ we have $d^{3} \Phi=0$ so we have the simplification to

$$
g_{y^{3}}\left(0, \nu_{k}^{*}\right)=\left\langle e, 3\left(e w_{y^{2}}\left(0, \nu_{k}^{*}\right)\right)^{\prime}\right\rangle=\left\langle e, \frac{1}{4}(\cos (3 \beta \xi)+\cos (\beta \xi) \cos (2 \beta \xi))\right\rangle=\frac{1}{8} .
$$

Since $G(y, w(y, \nu), \nu) \equiv 0$, then $P \Phi_{\nu}\left(0, \nu_{k}^{*}\right)=0$ which means the last equality of (7) yields $w_{\nu}\left(0, \nu_{k}^{*}\right)=0$, thus $g_{\nu}\left(0, \nu_{k}^{*}\right)=0$. Finally, $d \Phi_{\nu} \xi=\xi^{\prime \prime \prime \prime}$, implies $\operatorname{Pd} \Phi_{\nu}\left(0, \nu_{k}^{*}\right)(e)=\beta^{4} P(e)=0$ and $d^{2} \Phi\left(0, \nu_{k}^{*}\right)(e, 0)=0$ by the formula for the second derivative. This leads to $w_{y \nu}\left(0, \nu_{k}^{*}\right)=0$ and we obtain

$$
g_{y \nu}\left(0, \nu_{k}^{*}\right)=\left\langle e, e^{\prime \prime}\right\rangle=-\beta^{2} .
$$

Thus, we have $g\left(0, \nu_{k}^{*}\right)=g_{y}\left(0, \nu_{k}^{*}\right)=g_{y y}\left(0, \nu_{k}^{*}\right)=g_{\nu}\left(0, \nu_{k}^{*}\right)=0$ and $g_{y y y}\left(0, \nu_{k}^{*}\right) g_{y \nu}\left(0, \nu_{k}^{*}\right)<$ 0 . By standard results from singularity theory this indicates a supercritical pitchfork bifurcation Golubitsky and Schaeffer, 1985]. For the bifurcation point at $\nu_{1 / 2}^{*}$, this automatically guarantees that the bifurcating branches of steady-states are asymptotically stable.

\section{Numerical continuation}

Having determined that branches of equilibria with (hidden) symmetry emanate from the zero solution in supercritical pitchfork bifurcations, we turn our attention to the global behaviour of these solutions. Diagram 1 shows the primary branches with wave number $k$ equal to $1 / 2$ and $3 / 2$ in blue and to 1 and 2 in red. All bifurcation points at which primary branches lose - or gain - stability are included and the corresponding secondary branches are shown in green. Stable solutions are shown with solid lines and 
unstable solutions with dashed lines. The upper and lower parts of the primary branches corresponding to wave numbers $1 / 2$ and $3 / 2$ are related by the reflection symmetry $\kappa$ and thus display the same bifurcations.

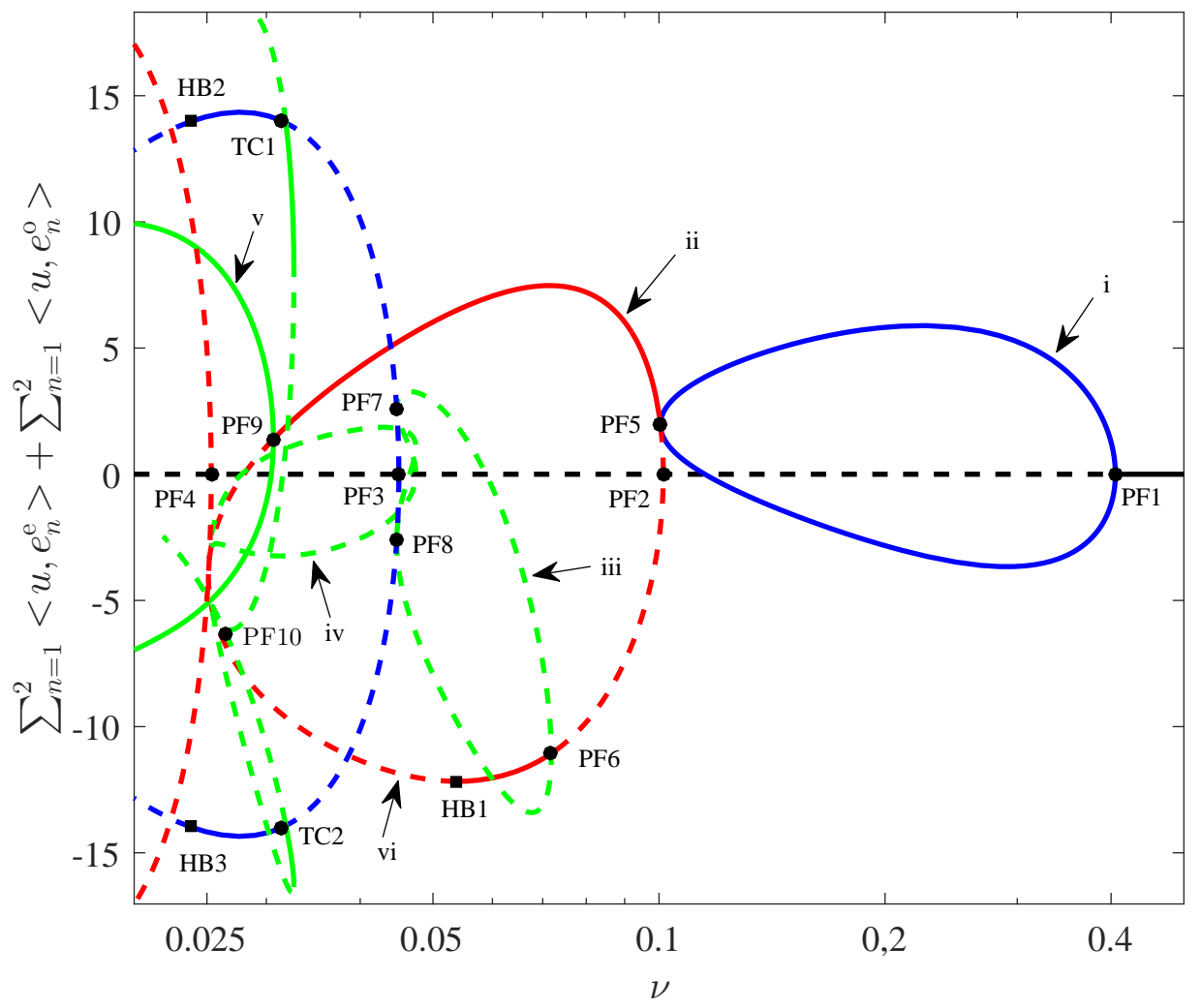

Fig. 1. Partial bifurcation diagram of the KS IBVP for homogeneous Dirichlet boundary conditions. Shown are the primary branches emanating at $\nu=1 /(\pi k)^{2}$ for $k=1 / 2,3 / 2$ in blue and for $k=1,2$ in red, and secondary branches bifurcating off the latter in green. Solid lines denote stable branches and dashed lines denote unstable branches. Pitchfork bifurcations are denoted by solid circles and Hopf bifurcations by solid squares. On the vertical axis the sum of projections onto the kernel functions $e_{n}^{\mathrm{e}}$ and $e_{n}^{\mathrm{o}}(n=1,2)$ is shown. The Roman numerals $\mathbf{i}-\mathbf{v i}$ correspond to the solutions visualized in Figure 4

The branch with $k=1 / 2$ is entirely stable and connected to the branch with $k=1$ at pitchfork bifurcation $\mathrm{PF}_{5}$. The top branch of the $k=1$ family turns stable here, and spawns a stable branch of equilibria without reflection symmetry in $\mathrm{PF}_{9}$. The bottom branch of this family also turns stable in a pitchfork bifurcation, $\mathrm{PF}_{6}$, and subsequently produces a stable time-periodic solution in Hopf bifurcation $\mathrm{HB}_{1}$. The branch with $k=3 / 2$ turns stable in a transcritical bifurcation, as expected given the symmetry considerations explained in Section 3, and then exhibits the second Hopf bifurcation around $\nu=0.025$. Around this parameter value, two symmetry-related equilibria on the above-mentioned stable secondary branch co-exist with two symmetry-related stable equilibria on the $k=3 / 2 \mathrm{branch}$, or two stable timeperiodic solutions. The primary branch from $\nu_{k}^{*}$ with $k=2$ is unstable down to $\nu=0.02$, where we stopped the continuations.

Details of the bifurcation diagram around the Hopf bifurcations are shown in Figures 2 and 3 . The periodic solutions originating from $\mathrm{HB}_{1}$ inherit a discrete symmetry from the $k=1$ branch, namely a shift over half the period followed by reflection $\kappa$. This symmery is broken in pitchfork bifurcation PPF. The branch of periodic solutions from $\mathrm{HB}_{2}$ has no symmetry. Both branches of periodic solutions undergo period doubling bifurcations that could signal the onset of more complicated spatio-temporal behaviour, beyond the scope of the current paper. 
Along the various branches of equilibria and periodic orbits we have visualized a number of solutions. They are shown in Figure 4. The spatial structure of the solutions at these relatively large values of the viscosity is dominated by wave numbers up to two, and the amplitude increases steadily with decreasing viscosity.

\section{Conclusion}

Using Lyapunov-Schmidt reduction and numerical continuation, and exploiting the hidden symmetry, we have given a fairly complete overview of the bifurcation structure of the KS equation with Dirichlet BC near the onset of nontrivial behaviour. In confluence with earlier results by $\mathrm{Li}$ and Chen [2001], this overview includes a complete description of all bifurcations from the zero solution. These are all supercritical pitchfork bifurcations, but come in two families: one breaks the reflection symmetry of the system under consideration, while the other breaks a reflection symmetry of an extended problem with periodic boundary conditions and is thus due to hidden symmetry.

Using numerical continuation, we computed the first four primary branches, the bifurcation points at which these lose stability and the resulting secondary branches. Steady-state bifurcations from the primary branches can be of the pitchfork and transcritical type, and both are explained in terms of hidden symmetry. The secondary branches include time-periodic solutions that exhibit pitchfork as well as period doubling bifurcations. The resulting partial bifurcation diagram, shown in Figure 1, is as complicated as one might expect from such a highly nonlinear system, and includes a wide range of parameters in which several stable equilibria and periodic solutions co-exist.

In addition to elucidating the transition from trivial to spatio-temporally structured behaviour in the KS problem with Dirichlet boundary conditions, we have, with this study, provided a neat example of the consequences of hidden symmetry. This phenomenon has mostly been demonstrated through evolution equations of the reaction-diffusion type, for instance by Crawford et al. [1991] and Golubitsky and Stewart 2002]. We hope that the KS example will prove a useful addition to literature on hidden symmetry and aid in future studies of parabolic initial-boundary value problems.

\section{Acknowledgments}

EF was supported by an Ontario Graduate Scholarship, PLB and LvV are supported by the National Science and Engineering Research Council of Canada in the form of a Discovery Grant.

\section{Appendix A Numerical simulation and continuation}

The continuation and stability analysis presented in Section [5] is based on the numerical simulation of IBVP (12). Details of the discretization scheme can be found in van Veen [2016], here we present only its extention to the linearized equations and the application of Newton-Krylov and Arnoldi iteration.

The simulation algorithm is based on that of Rothe [1930]: first, time is discretized and then a linear BVP is solved for each time step with the aid of Green's function. Since we only need to integrate the equations over short time intervals for the purpose of continuation of equilibria and periodic orbits, the semi-implicit Euler method is adequate for time discretization. The BVP to be solved in each time step is then

$$
\begin{aligned}
& \mathcal{L} u^{(k+1)} \equiv\left(1+h \partial_{x}^{2}+h \nu \partial_{x}^{4}\right) u^{(k+1)}=u^{(k)}-\frac{h}{2}\left(u^{(k)}\right)_{x}^{2} \\
& u^{(k+1)}(-1)=u_{x x}^{(k+1)}(-1)=u^{(k+1)}(1)=u_{x x}^{(k+1)}(1)=0 .
\end{aligned}
$$

and perturbing $u$ by $w$ and $\nu$ by $\omega$, we find the linearized equation

$$
\begin{gathered}
\mathcal{L} w^{(k+1)}=w^{(k)}-h\left(u^{(k)} w^{(k)}\right)_{x}-h w u_{x x x x}^{(k+1)} \\
w^{(k+1)}(-1)=w_{x x}^{(k+1)}(-1)=w^{(k+1)}(1)=w_{x x}^{(k+1)}(1)=0 .
\end{gathered}
$$


where $h$ is the time step size. The solution is given in terms of Green's function for the differential operator $\mathcal{L}$ with the given boundary conditions as

$$
\begin{aligned}
u^{(k+1)} & =G * u^{(k)}+\frac{h}{2} D G *\left(u^{(k)}\right)^{2} \\
w^{(k+1)} & =G *\left(w^{(k)}-h w u_{x x x x}^{(k+1)}\right)+h D G *\left(u^{(k)} w^{(k)}\right)
\end{aligned}
$$

where the asterisk denotes the spatial convolution. Finally, the fourth derivative of the solution is time stepped according to

$$
u_{x x x x}^{(k+1)}=D^{4} G * u^{(k)}+\frac{h}{2} D^{5} G *\left(u^{(k)}\right)^{2}
$$

Green's function is known analytically, so the only numerical approximation is a quadrature rule for approximating the convolutions. Following the approach of van Veen [2016], we represent the solution on a global, closed Chebyshev grid and employ an exponentially accurate combination of barycentric interpolation and Clenshaw-Curtis quadrature. For $\nu>0.03$, we used a grid order of $N=32$ and below that we set $N=64$, while the time step was fixed to $h=10^{-3}$. Several solutions along each of the curves were recomputed at double their resolution and smaller time step for verification.

Following the approach explained in detail by Sánchez Umbría and Net [2016], the time-steppers for the IBVP and its linearization are all that is needed for the continuation and stability analysis of equilibria and periodic orbits. Hence, we need only state briefly the continuation equations, tolerances and convergence properties of the algorithm.

The continuation equations are best formulated in terms of the flow of the vector of grid point values $\boldsymbol{u} \in \mathbb{R}^{N+1}$, denoted by $\phi(\boldsymbol{u}, t, \nu)$. The equations to solve for equilibria and periodic orbits are

$$
\phi(\boldsymbol{u}, P, \nu)-\boldsymbol{u}=0
$$

and a phase condition $\psi(\boldsymbol{u}, P)=0$, namely

$$
\psi \equiv P-c=0 \text { (for equilibria), or } \psi \equiv u_{n / 2}=0 \text { (for periodic orbits) }
$$

where $c=1$ is an integration time long enough to pre-condition the linear system for the Newton-Raphson update step. We compute branches of solutions to BVP (A.8 A.9) using pseudo-arclength continuation. The residual thresold is fixed to $10^{-8}$ in the norm

$$
\|(\boldsymbol{u}, P, \nu)\|=\sqrt{\|\boldsymbol{u}\|_{2}^{2}+\psi^{2}}
$$

where $\|\cdot\|_{2}$ denotes the approximate $L_{2}-$ norm of a function as obtained by Clenshaw-Curtis quadrature on the grid. We obtain quadratic convergence of the Newton-Raphson iterates with about 10 Krylov subspace iterations and a tolerance of $10^{-6}$ for the linear solving.

\section{References}

Crawford, J. D., Golubitsky, M., Gomes, M. G. M., Knobloch, E. and Stewart, I. N. [1991] Boundaryconditions as symmetry constriants in Lecture Notes in Mathematics, 1463, p. 63, Springer-Verlag, New-York.

Cvitanovic, P. and Davidchack, R.L. and Siminos, E. [2010] "On the state-space geometry of the KuramotoSivashinsky flow in a periodic domain". SIAM Appl. Dyn. Syst., 9, p. 1.

Galaktionov, V. A., Mitidieri, E. and Pohozaev, S. I. [2008] "Existence and non-existence of a global solution to the Kuramoto-Sivashinsky equation," Doklady Math., 77, p. 238.

Golubitsky, M. and Schaeffer, D. G. [1985] Singularities and Groups in Bifurcation Theory (Vol. 1), Springer, New-York.

Golubitsky, M., Stewart, I. and Schaeffer, D. G. [1988] Singularities and Groups in Bifurcation Theory (Vol. 2), Springer, New-York.

Golubitsky, M. and Stewart, I. [2002] The symmetry perspective: from equilibrium to chaos in phase space and physical space, Birkhäuser, Basel. 
Kuramoto, Y. and Tsuzuki, T. [1976] "Persistent propagation of concentration waves in dissipative media far from thermal equilibrium," Prog. Theor. Phys., 55, p. 356.

Li, C. and Chen, G. [2001] "Bifurcation analysis of the Kuramoto-Sivashinsky equation in one spatial dimension," Int. J. Bifurc. Chaos 11, p. 2493.

Misbah, C. and Valence, A. [1994]. "Secondary instabilities in the stabilized Kuramoto-Sivashinsky equation," Phys. Rev. E, 49, p. 166.

Rothe, E. [1930] "Zweidimensionale parabolische Randwertaufgaben als Grenzfall eindimensionaler Randwertaufgaben," Math. Ann. 102, p. 650.

Sánchez Umbría, J. and Net, M. [2016] "Numerical continuation methods for large-scale dissipative dynamical systems," Eur. Phys. J. - Spec. Top., in press.

Sivashinsky, G. I. [1977] "Nonlinear analysis of hydrodynamical instability in laminar flames - I. Derivation of basic equations," Acta Astron., 4, p. 1177.

van Veen, L. [2016] "On Green's function-based time stepping for semilinear initial-boundary value problems," J. Comp. Appl. Math. 308, p. 46.

Wittenberg R. and Holmes, P. [1999] "Scale and space localization in the KuramotoSivashinsky equation," Chaos, 9, p. 452.

Zhang, Y. and Song, L. and Axia, W. [2011] "Dynamical bifurcation for the Kuramoto-Sivashinsky equation," Nonlinear Analysis, 74, p. 1155. 


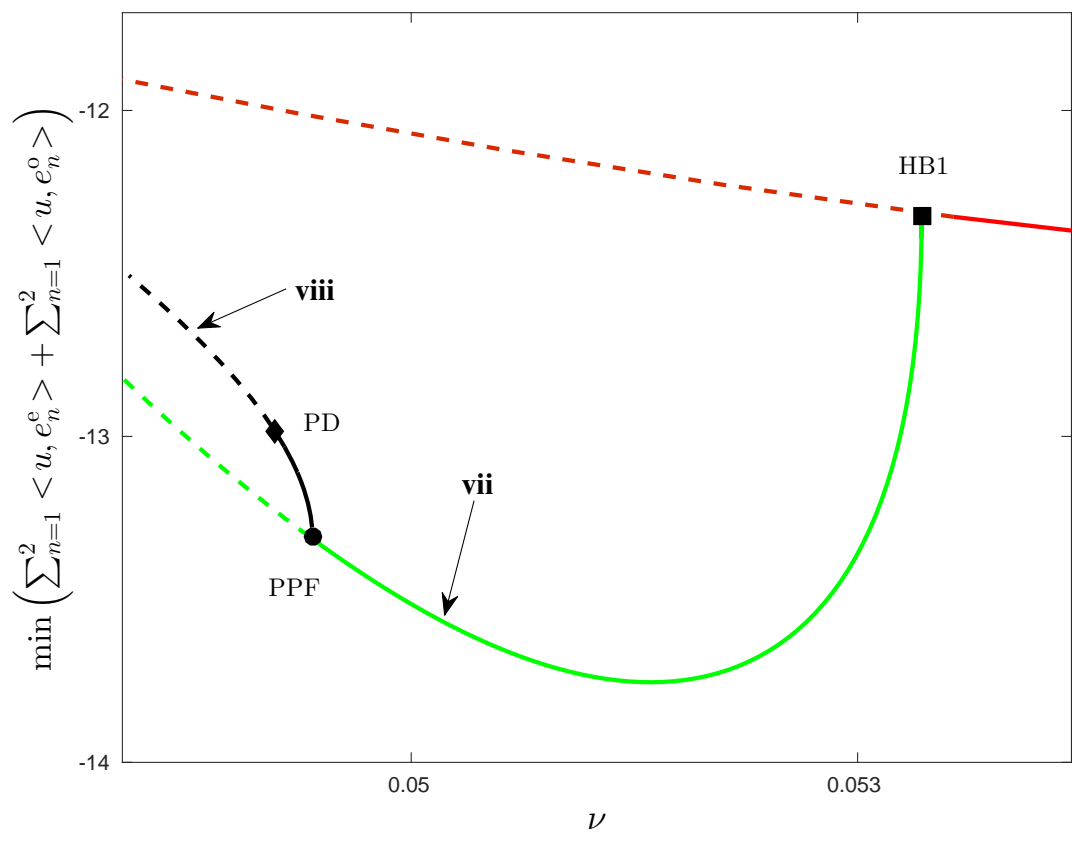

Fig. 2. Detail of diagram 1 around HB1. PPF denotes a pitchfork birucation of a periodic orbit and the solid diamond denotes a period doubling bifurcation. The roman numerals vii-viii correspond to the solutions visualized in Figure 4

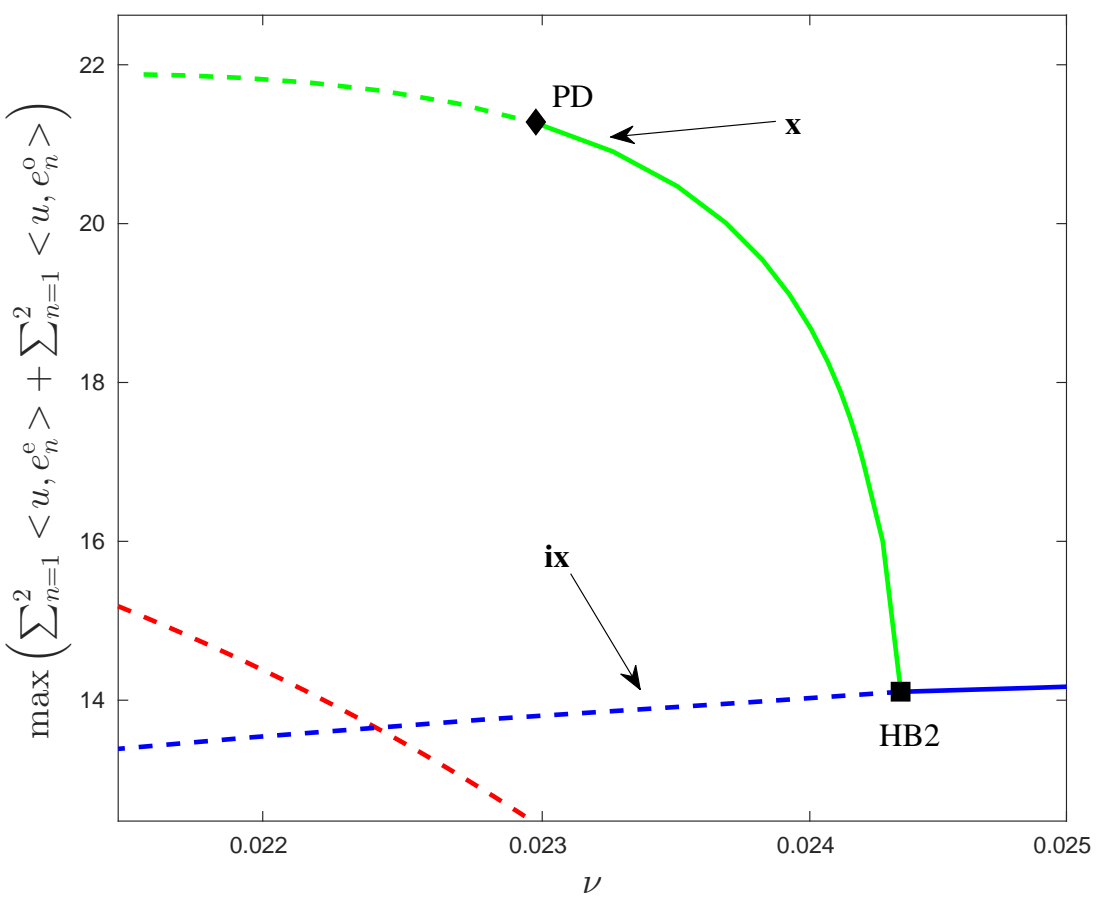

Fig. 3. Detail of diagram 1 around HB2. The solid diamond denotes a period doubling bifurcation. The roman numerals $\mathbf{i x}-\mathbf{x}$ correspond to the solutions visualized in Figure 4 

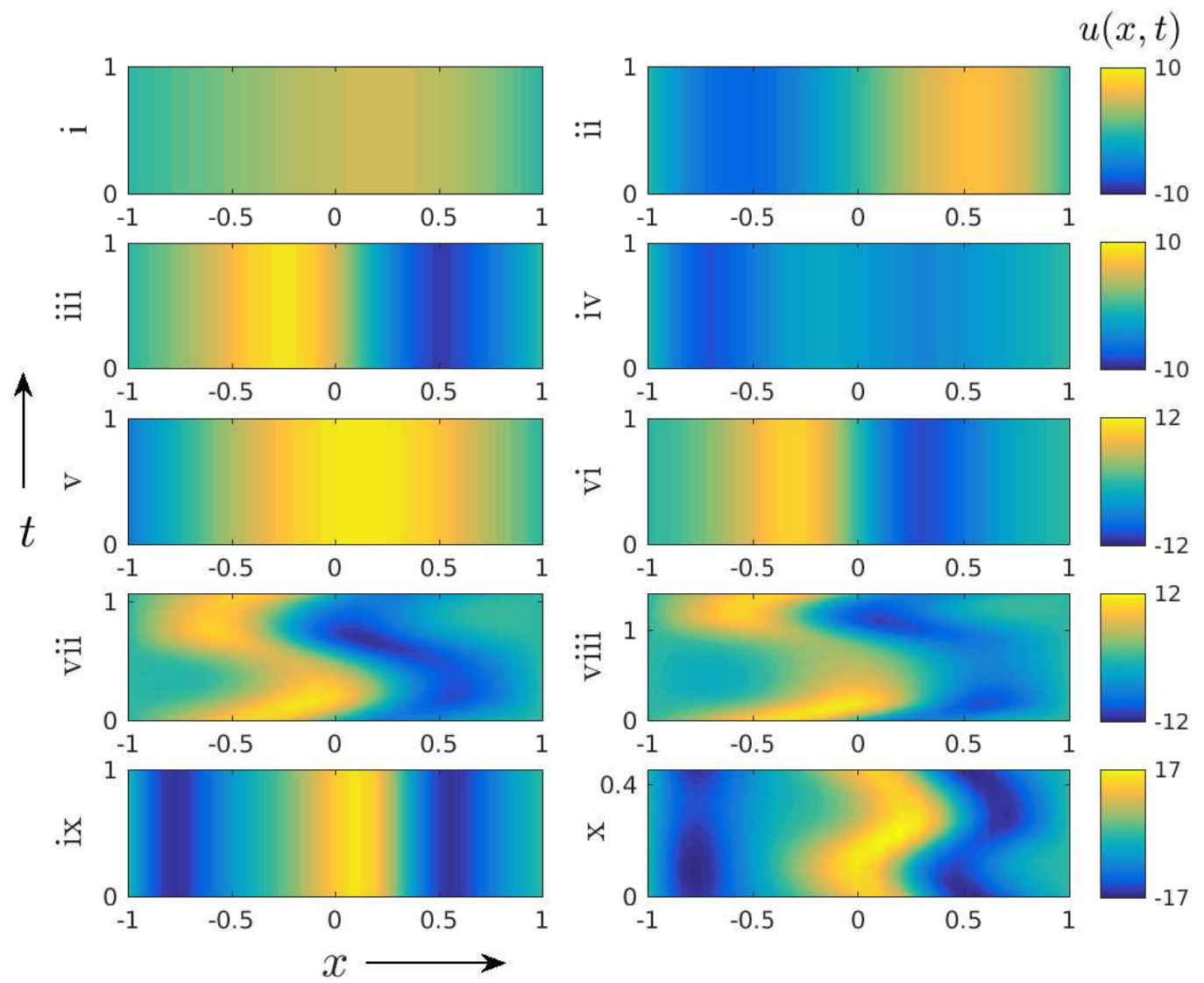

Fig. 4. Some solutions corresponding to the labels in Figures 13. The horizontal axes denote space and the vertical axes denote time. The solutions $u(x, t)$ are shown using the colour scale on the right hand side of each row. 